$$
\text { NASA-CR-122301 page26" }
$$

\title{
CHARACTERISTICS OF MAGNETOSHEATH PLASMA OBSERVED AT LOW ALTITUDES IN THE DAYSIDE MAGNETOSPHERIC CUSPS
}

J. DAVID WINNINGHAM

Atmospheric and Space Sciences

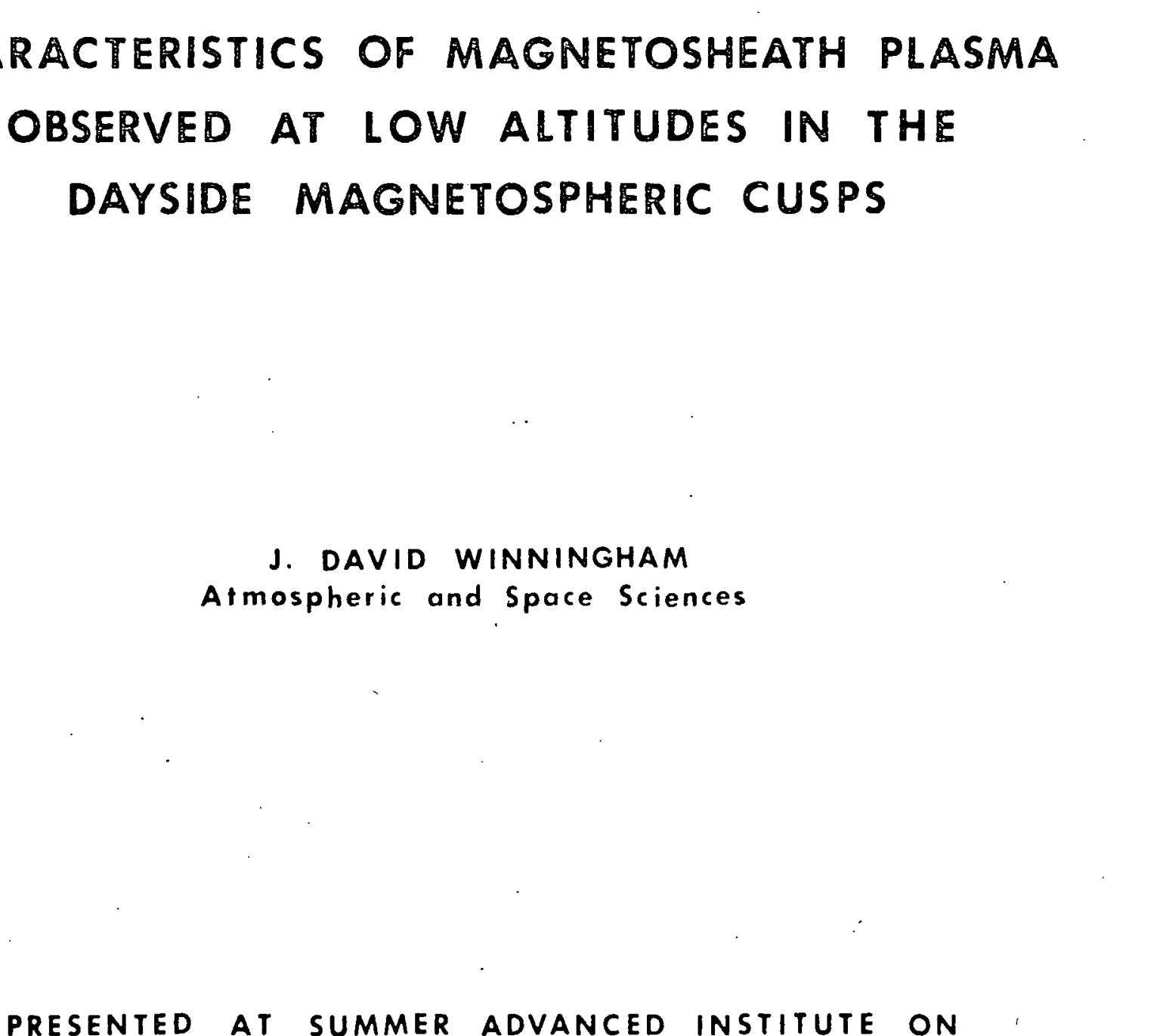

PRESENTED AT SUMMER ADVANCED INSTITUTE ON EARTH'S PARTICLES AND FIELDS AUGUST 20 - SEPTEMBER 10,1971 CORTINA, ITALY

THIS WORK WAS SUPPORTED BY NASA CONTRACT NAS 5-9112

$$
\text { JULY 7, 1971 }
$$

THE UNGYERSTY OF TEXAS AT DALLAS 
CHARACTERISTICS OF MAGNETOSHEATH PLASMA OBSERVED AT LOW

ALTITUDES IN THE DAYSIDE MAGNETOSPHERIC CUSPS

\begin{abstract}
Magnetosheath plasma penetrating to low altitudes in the dayside cusp region of the magnetosphere has been observed by the ISIS 1 soft particle spectrometer (SPS). The extent of these particle fluxes in local magnetic time and invariant latitude, their vartation with magnetic activity, and their pitch angle distribution are given. Comparison between the SPS data and energetic particle data indicates that the boundary between open and closed field lines on the dayside is associated with a sharp drop in the outer zone $>1 \mathrm{kev}$ electron fluxes. It is shown that these newly identified cusp fluxes provide the necessary energy to produce observed dayside auroral oval phenomena.
\end{abstract}

\title{
INTRODUCTION
}

Heikkila et al (1970), Winningham (1970) and Heikkila and Winningham (1971) gave the first definitive evidence for linking dayside "soft zone" fluxes with a magnetosheath source via the cusps in the dayside magnetosphere. The existence of magnetosheath plasma penetration to low altitudes had long been postulated by theoreticians and experimentalists alike (the reader is referred to the review in Winningham (1970) and Heikkila and Winningham (1971)). This paper will extend the dayside results presented In the earlier ISIS 1 papers. 
Winningham (1970) identified the invariant latitude, denoted by $\Lambda_{C L}$, where outer zone fluxes cease and soft fluxes with magnetosheath characteristics begin as the last closed field line on the dayside. He further postulated. this boundary to mark the beginning of interconnected terrestrial and interplanetary field lines. The Invariant latitude, $\Lambda_{\mathrm{CU}}$, where soft magnetosheath-1ike proton fluxes end was identified as marking the last merged field line which has free access to magnetosheath plasma. Electron fluxes are often observed above $\Lambda_{C U}$ but they do not, in general, exhibit magnetosheath characteristics, and no protons are observed in the polar cap region. This terminology and its implied assumptions will be used in the remainder of this paper.

\section{INSTRUMENTATION}

ISIS 1 was launched into a 570 by $3500 \mathrm{~km}$ polar orbit (inclination $88.5^{\circ}$ ) on 30 January 1969 . The ISIS 1 soft particle spectrometer (SPS) simultaneously measures the differential energy spectra of positive and negative particles in the energy range $10 \mathrm{ev}$ to $12 \mathrm{kev}$ per unit charge with a resolution of $\pm 40 \%$ (see Heikkila, et al., 1970 and Winningham, 1970 for a more detailed description). Results presented in this paper were obtained with a $15^{\circ} \times 35^{\circ}$ collimator pointed perpendicular to the satellite spin axis.

The results obtained in the swept mode of operation areconveniently displayed as energy-time spectrograms. Each differential energy sweep of the instrument is shown as a separate line in the spectrogram (top portion of Figure $1 \mathrm{a}$ and $\mathrm{b}$ ) with the density of the trace being modulated by the counting rate. A readout of 15 or less per sample is inhibited, with accumulation continuing during successive sampling intervals until >15 
counts are accumulated. The middle and lower graphs of the spectrogram give the total number and energy flux over the $10 \mathrm{ev}$ to $12 \mathrm{kev}$ energy range. OBSERVATIONS IN THE DAYSIDE HIGH-LATITUDE REGION

A pair of high resolution spectrograms (one spectrum every $1 / 2 \mathrm{sec}$ or $4 \mathrm{~km}$ ) are reproduced in Figure $1 \mathrm{a}$ and $\mathrm{b}$. This pass, which began at 19:50:53 UT on 28 April 1969, occurred during the recovery phase $\left(K_{p}=3^{-}\right)$of a storm that commenced at $\sim 300$ hours UT on the same day. The magnetic local time was 1645 hours. At the beginning of this pass only low-energy electrons are observed in the polar cap region. The vertical bars appearing at 20 second intervals in the spectrogram are due to solar UV contamination. An isotropic flux of protons begins at 19:51:30 UT $\left(\Lambda_{\mathrm{CU}}=76.5^{\circ}\right)$ and continues to 19:53:26 UT $\left(\Lambda_{\mathrm{CL}}=73^{\circ}\right)$. Below $\Lambda_{\mathrm{CL}}$ the proton flux peaks at large pitch angles. Between $\Lambda_{C L}$ and $\Lambda_{C U}$ an isotropic flux of electrons with a spectral peak at $\sim 100 \mathrm{ev}$ is observed. Below $\Lambda_{\mathrm{CL}}$ the electron average energy increases, and the pitch angle distribution becomes anisotropic towards $90^{\circ}$.

It should be noted that electron fluxes for most cusp passes exhibit a greater variability than shown in Figure la (see Winningham (1970) for a larger collection of spectrograms). This pass was selected because of the large pitch angle scan and good angular resolution, not because it is "the typical pass."

Representative spectra from the cusp data in Figure $1 a$ and $b$ are given in Figure 2. The cusp electron spectrum at a pitch angle $\left(\alpha_{p}\right)$ of $29^{\circ}$ is observed to have a peak at $100 \mathrm{ev}$ as do a majority of the cusp spectra observed with ISIS 1 (Figure 3). Below $\sim 60$ ev a roughly power law component is observed in both cusp and outer zone spectra which is due to atmospheric photoelectrons (He1kkila, 1970) and secondaries. Outer 
zone electron spectra (Figure 2) gradually harden from an average energy of $\sim 500 \mathrm{ev}$ just below $\Lambda_{\mathrm{CL}}$ to $\sim 1 \mathrm{kev}$ when they go below threshold at $\Lambda=68^{\circ}$. Just below $\Lambda_{C L}$ the electron flux is isotropic but rapidly becomes anisotropic towards $90^{\circ}$ as the invariant latitude decreases. Proton spectra observed in the cusp region peak at $~ 600$ ev (Figure 2) with a decrease in intensity towards higher and lower energies. Below $\Lambda_{C L}$ the proton flux decreases in intensity, becomes harder, and is peaked at $90^{\circ}$ pitch angle.

Rather than compare the spectra in Figure 2 with those in the magnetosheath for different periods as was done by Heikk1la and Winningham (1970), comparison will be made in a later section between near concurrent IMP 5 (Frank, 1970) and ISIS 1 spectra recorded on 11 July 1969.

Close inspection of detailed spectral printouts reveals that up to 19:53:26 UT $\left(\Lambda=73^{\circ}\right)$ electron spectra are identical to the cusp spectrum In Figure 2, and those after 19:53:36 (below $\Lambda=72.9^{\circ}$ ) are similar to the outer zone spectra in Figure 2. In the intervening region (10 seconds) the spectra appear to be an admixture of both. Burrows (private communication, indicates that above $\Lambda=71.6^{\circ}$ the trapped, energetic (>20 kev) electron fluxes begin a rapid drop to background. The $>20 \mathrm{kev}$ flux reaches $10 \%$ of maximum at $72.3^{\circ}$ and background at $72.6^{\circ}$. The $>200 \mathrm{kev}$ detector (which has a larger geometric factor) reaches background at $\Lambda=72.8^{\circ}$. It thus appears that on this pass, hard outer zone electron fluxes extend up to the boundary between softer outer zone electrons and magnetosheath electrons. Winningham (1970) compared a larger number of passes and found similar results to the above. However, during very active periods fluxes of $>20 \mathrm{kev}$ electrons can be above background between $\Lambda_{\mathrm{CL}}$ and $\Lambda_{\mathrm{CU}}$. Further intercomparison is being made and the results will be presented in a future paper. 
PITCH ANGLE DISTRIBUTION

In the normal mode of SPS operation (one spectrum every two seconds) only three samples are obtained in one quadrant resulting in a coarse pitch angle distribution. In the all-radial sweep mode approximately 12 samples (one sweep every half second) are obtained in one quadrant. Figure 4 illustrates a typical high resolution electron and proton pitch angle distribution for the cusp fluxes shown in Figure $1 \mathrm{a}$ and $\mathrm{b}$. As noted earlier soft electron and proton fluxes from the dayside cusp are observed from. $\Lambda_{\mathrm{CL}}=73^{\circ}$ to $\Lambda_{\mathrm{CU}}=76.5^{\circ}$. During this period the pitch angle range scanned was $90^{\circ} \pm 71^{\circ}$ at $\Lambda=78^{\circ}$ to $90^{\circ} \pm 80^{\circ}$ at $\Lambda=73^{\circ}$. The large depressions in number and energy fluxes (see Figure la and b) occur when the instrument scans into the loss cone for upcoming particles (the large regulz.r spikes are sun pulses).

Figure 4 shows that the number and energy flux for the primary electron beam (73 to $420 \mathrm{ev}$ ) in the cusp are isotropic up to $\alpha_{p}-135^{\circ}\left(\alpha_{p}\right.$ is the pitch angle for the normal to the detector aperture). Above $135^{\circ}$ both the number and energy flux exhibit the same relative decrease in value. A particle at $\alpha_{p}=135^{\circ}$ and $140^{\circ}\left(45^{\circ}\right.$ and $40^{\circ}$ incident angles) will mirror at 700 and $200 \mathrm{~km}$ respectively (see Figure 4 for a graph of mirror heights appropriate to the altitude of the results presented in Figure 4). Particles incident at angles less than $38^{\circ}\left(>142^{\circ}\right.$ return angle) will find thei: mirror points below $100 \mathrm{~km}$. Thus particles observed above - $140^{\circ}$ cannot be particles that have simply mirrored below the satellite.

Figure 5 gives electron spectra for pitch angles just prior to and after the decrease in Figure 4. The spectra at $\alpha_{p}=144^{\circ}$ and $151^{\circ}$ are observed to be similar in shape to the one at $135^{\circ}$ but decreased in amplitude. This 
seems to indicate that a significant amount of elastic scattering exists In the ionosphere below the cusp. As the pitch angle increases, the peak In the $>73 \mathrm{ev}$ range is lost and the spectra appear to be due to backscatter of secondaries and degraded primaries. The return flux for particles inside the loss cone is observed to be much smaller for soft outer zone fluxes (see Figure la at 19:53:38 UT). As mentioned earlier the average energy for the soft outer zone spectra is 5 to 10 times that of the cusp spectra. The 500 ev outer zone electrons thus deposit a greater fraction of their incident energy in the ionosphere as compared to the $100 \mathrm{ev}$ cusp electrons. This difference in the fraction of the incident energy flux lost (i.e. different albedos) can probably be explained by the manner in which energy is lost as a function of incident particle energy. The $-100 \mathrm{ev}$ cusp electrons begin their energy loss at very high altitudes $(-600 \mathrm{~km})$ whereas the - $500 \mathrm{ev}$ outer zone electrons begin theirs at $\sim 400 \mathrm{~km}$ (Rees, 1964). If at higher altitudes processes such as coulomb scattering off heavy ions, collisional excitation, or weak wave-particle interaction dominate over Ionization then large angular scattering is possible without large energy losses. This would result in a large albedo electron flux with a spectrum similar to the incident spectrun (see figure 5). As the altitude of the energy loss region decreases (i.e. increasing particle energy) ionization, with its accompanying large incremental energy loss, will probably become the major loss mechanism. If present, the albedo flux would be composed mainly of highly degraded primaries and secondaries bearing little resemblance to the incident spectrum. This is consistent with the differences observed in cusp and outer zone albedo fluxes in Figure 1a. 
An alternative explanation to the above would be an electrostatic double layer in the cusp below the satellite. The parallel electric field of the double layer would raise the mirror heights and thus decrease the energy loss. Assuming a $100 \mathrm{~V}$ potential to exist between the satellite $(2600 \mathrm{~km})$ and the $100 \mathrm{ev}$ maximum loss region $(-300 \mathrm{~km})$, a field of $\sim 50 \mu \mathrm{v} / \mathrm{m}$ would result. This value is not prohibitively large but has one drawback. Such a field would accelerate ionospheric electrons into the energy range of ISIS 1. No such fluxes are observed on 28 April 1969 or other ISIS 1 passes which scan from 0 to $180^{\circ}$ pitch angle.

Figure 4 indicates that the loss process for protons occurs in the altitude range below $\sim 700 \mathrm{~km}\left(\alpha_{\mathrm{p}}=135\right)$. The albedo proton flux above $135^{\circ}$ is observed to be vanishingly small. This is consistent with the usual assumption of loss from the primary proton beam by charge exchange (i.e. conversion to hydrogen which is not measured by the SPS even if it is backscattered).

Assuming that both electron and proton angular distributions are isotropic over the upper hemisphere $\left(10^{\circ} \leq \alpha_{p} \leq 170^{\circ}\right.$ in the cusp region), the fraction of the incident energy that is lost can be calculated. For protons In Figure $1 \mathrm{~b}$ it is obvious (Figure 4) that all the incident primary energy $\left(1.0 \times 10^{-1} \mathrm{ergs} / \mathrm{cm}^{2} \mathrm{sec}\right)$ is deposited in the ionosphere. Using an average energy of $\sim 1 \mathrm{kev}$ and the results of Eather (1967) this eneigy loss corresponds to $-5 \mathrm{R}$ of $\mathrm{HB}$ which is in good agreement with recent airborne measurements of the dayside aurora (Eather \& Mende, 1971a). According to Figure 4 approximately $60 \%$ the incident primary electron energy flux of $2.5 \times 10^{-1}$ ergs $/ \mathrm{cm}^{2} \mathrm{sec}$ is deposited in the ionosphere. Again these results are seen to be in quantitative agreement with Eather and Mende's (1971a) inference 
that particles causing distinct dayside aurora deposit $\sim 1.3 \times 10^{-1} \mathrm{ergs} / \mathrm{cm}^{2}$ $\sec ^{-1}$ into the Ionosphere and have an average energy of $\sim 100$ to $200 \mathrm{ev}$ (see Figure 3).

EXTENT OF MAGNETOSHEATH PLASMA PENETRATION AND ITS DEPENDENCE ON $\mathrm{K}_{\mathrm{p}}$ As pointed out earlier $\Lambda_{C L}$ is defined empirically as the boundary between hard, structureless outer zone fluxes and softer, structured cusp fluxes. Physically $\Lambda_{\mathrm{CL}}$ is the last closed magnetic field line on which significant bounce motion between hemispheres can be maintained (i.e. closed on the dayside of the magnetosphere). Between $\Lambda_{\mathrm{CL}}$ and $\Lambda_{\mathrm{CU}}$ (the upper limit of cusp proton fluxes) the ISIS 1 data indicates that terrestrial field lines have continuous free access to magnetosheath plasma. Figure 6 illustrates the extent of this region of magnetosheath plasma penetration In magnetic local time ( 8 to 16 hours). Data for a given hour interval is averaged and plotted at the mid-point of the interval. The largest sampling density is in the forenoon, $k_{p} \leq 3$ region with less statistical accuracy for other points. The lower limit of penetration, $\Lambda_{C L}$, is observed to be largest at local magnetic noon with a decrease before and after midday. Also $\Lambda_{C L}$ is observed to move progressively equatorward with increasing $K_{p}$. The upper limit, $\Lambda_{\mathrm{CU}}$, of cusp fluxes is observed to be less responsive to changes in $K_{p}$ (it should be noted, however, that due to orbit parameters the sampling density for $\Lambda_{C U}$ is much less than $\Lambda_{C L}$ ). Also $\Lambda_{C U}$ does not exhibit the same statistical magnetic time dependence as $\Lambda_{\mathrm{CL}}$. For some passes at increased $\mathrm{K}_{\mathrm{p}}, \Lambda_{\mathrm{CU}}$ is observed to track $\Lambda_{\mathrm{CL}}$ (i.e. the whole cusp moves equatorward without an appreciable change in width). A comparison with changes in solar wind and interplanetary magnetic conditions would 
probably be more appropriate than the above comparison to $k_{p}$. Such a comparison is presently being undertaken and will be reported in a future paper.

The above results do not imply that softer fluxes of electrons and protons do not exist before and after 8 and 16 hours magnetic local time. Significant fluxes of low energy particles are indeed present outside these limits but their spectra peak at higher energies and do not in general exhibit magnetosheath characteristics.

For magnetically quiet periods the boundary (in MLT) of magnetosheath fluxes can be quite sharp. During days when the local time is $\sim 7$ to 8 (or 16 to 17 ) hours, the dipole wobble causes a large diurnal variation in magnetic local time. When the dipole tilt results in times inside the 8 to 16 hour magnetic time interval, magnetosheath fluxes are observed; and when MLT is outside this period, non-magnetosheath fluxes are observed. During more disturbed periods magnetosheath fluxes are observed as early as 0500 MLT and as late as 1800 MLT as evidenced by Figure 1.

Using the results of Fairfield (1968) the 8 to 16 hour magnetic time interval at ISIS 1 altitudes maps into the magnetic equatorial plane at $~ 06$ and 18 hours local time (i.e. solar dawn-dusk). Thus if we take these results at face value, magnetosheath plasma has access to the magnetosphere across its entire front side during quiet periods and over a larger extent during disturbed periods. It will be extremely interesting in terms of magnetospheric structure and dynamics if the above results inferred from low-altitude measurements are verified by a comprehensive in situ survey. 
COMPARISON WITH OTHER OBSERVATIONS OF DAYSIDE CUSP FLXUES

Winningham (1970) and Heikkila and Winningham (1971) compared their dayside high-latitude spectra with earlier magnetosheath spectra and Inferred that the magnetosheath is the source for the dayside "soft zone". This comparison obviously suffers in that the measurements compared are neither concurrent in time and meridian nor obtained for similar solar wind and geomagnetic conditions. Also Winningham's (1970) and Heikkila and Winningham's (1971) observations were obtained during the recovery phase of a magnetic storm where $K_{p}$ was $5+$ and $\Sigma K_{p}$ equalled $41+$. These results (mainly the latitudinal width of the cusp) could thus be construed as a transient phenomena occuring only during large storms. As pointed out earlier in this work and that of Winningham (1970) this is not the case, however.

Figure 7 details a comparison of near coincident electron spectra obtained with the high-latitude, high-inclination IMP 5 spacecraft (Frank, 1970) and the low-altitude ISIS I polar satellite on 11 July 1969. These observations were made within $\sim 2$ hours of universal and local time and during a relatively quiet period with $K_{p}=1, A_{p}=6$, and $\Sigma K_{p}=11$. The electron average energy is $\sim 60 \mathrm{eV}$ and the electron number and energy fluxes are lower than normal (presumably a result of the quieter solar wind conditions, $\mathrm{V}_{\mathrm{H}}+=$ $332 \mathrm{~km} / \mathrm{sec}$ and $\mathrm{N}_{\mathrm{H}^{+}}=3.7 \mathrm{~cm}^{-3}$ ). For the region of energy overlap the ISIS 1 cusp spectrum is observed to be in quantitative agreement with the magnetosheath and mid-altitude cusp spectra obtained concurrently by IMP 5. The outer zone electron spectra are also observed to be in quantitative agreement (the outer zone fluxes were also much lower here than during more disturbed periods). The width of the cusp was however at least $2.5^{\circ}$ even for these low $K_{p}$ and solar wind conditions. $\Lambda_{c U}$ and the actual 
width could not be determined because data transmission began within the cusp region. The above results are in agreement with other quiet-time ISIS 1 cusp data and indicate the large width to be a permanent feature. The proton directional number flux $\left(3 \times 10^{6} / \mathrm{cm}^{2}\right.$ ster sec) was also low for this cusp traversal and the proton average energy was low $(-300 \mathrm{ev})$. This is also quite likely a result of the quiet solar wind (and presumably magnetosheath) conditions during this period.

As discussed earlier fluxes of protons and electrons with magnetosheath characteristics are observed nearly continuously in a $2^{\circ}$ to $3^{\circ}$ zone (Figure 6) above the limit of closed field lines (evidenced by a large drop in the outer zone fluxes). Frank and Ackerson (1971) give two examples of very narrow $\left(20-30 \mathrm{~km}\right.$ or $\left.\Delta \Lambda=0.2^{\circ}\right)$ electron spikes obtained with INJUN 5 which they identify as the low-altitude signature of the cusp. At $\Lambda_{\mathrm{CU}}=76.5^{\circ}$ (19:51:30 UT) in Figure la a large increase in both. the number and energy flux is observed, but the average energy and spectral shape are simflar to the remainder of the cusp. This feature is three seconds $(\sim 21 \mathrm{~km})$ wide and is probably similar to the narrow $(20-30 \mathrm{~km})$ features observed by Frank and Ackerson (1971) (see discussion section). Other examples of this narrow feature have been observed in the ISIS 1 data (see Winningham, 1970) and are generally found close to either $\Lambda_{\mathrm{CL}}$ or $\Lambda_{\mathrm{CU}}$.

An apparent discrepancy also exists between the ISIS 1 and IMP 5 results. Frank (1970) observed 690 to $1100 \mathrm{eV}$ protons to lie polewaru of 305 to $510 \mathrm{ev}$ electrons in the mid-altitude cusp. In general no such separation is observed In the ISIS data and in particular no such separation is found in the data at 0900 UT on 11 July 1969.

Russell et al. (1970) reported a high-altitude observation of the northern dayside cusp at $45^{\circ}$ geomagnetic latitude during the large storm of 
October 31-November 1, 1968. During quiet periods the inclination of OGO 5 does not allow it to traverse the cusp. They concluded the cusp to be moving back and forth at velocities comparable to the satellite in response to changes in geomagnetic and solar wind parameters. During the storm of February 2-3, $1969 \Lambda_{\mathrm{CL}}$ was observed to move to as low as $67^{\circ}$ at 1000 hours local time with ISIS 1 . The cusp widths for two passes at $k_{p}=6^{-}$and 7 and low $\Lambda_{C L}$ during this period were no larger than usual indicating the varying response of the cusp to changes in $K_{\mathrm{p}} \cdot$.. During a small storm on 8 June 1969 the northern cusp was observed to be $8^{\circ}(\sim 2000 \mathrm{~km})$ wide at $3500 \mathrm{~km}\left(\Lambda_{\mathrm{CL}}=75.5^{\circ}, \mathrm{MLT}=0100\right.$ hours $)$ for a time when $\mathrm{K}_{\mathrm{p}}$ was $3^{-}\left(3^{-}\right.$ was also the maximum $\mathrm{K}_{\mathrm{p}}$ ). This would correspond to $\sim 800 \mathrm{~km}$ at auroral heights. As mentioned earlier in this paper and in Russel1 (1971) changes in the cusp are probably more intimately related to changes in the solar wind and interplanetary magnetic field than to changes in geophysical parameters.

\section{DISCUSSION}

The results presented in this paper and those of Frank (1970), Frank and Ackerson (1971), Heikkila and Winninghan (1971), Russel1 et al. (1971), and Winningham (1970) have established the existence of two cusp-like regions In the dayside magnetosphere and the penetration of magnetosheath plasma to low altitudes through them. The main difficulty in reconciling these various measurements lies in the width and structure of the cusp at auroral helghts. Frank (1970), using IMP 5 data indicates the low-altitude width should map to 20 to $30 \mathrm{~km}\left(\Delta \Lambda=0.2^{\circ}\right)$ at the ionosphere, and reports a feature of the INJUN-5 data (Frank and Ackerson, 1970). which would support this. On the other hand the ISIS 1 data indicates a region which is on the average 2 to $3^{\circ}$ wide at low altitudes. 
The discrepancy between the ISIS 1 data and the projection of the IMP 5 data onto the Ionosphere can be resolved, I believe, in the following way. Frank (1970) indicates the latitudinal width of the cusp to be $-1 R_{E}$ at its high altitude limit (which lies at $~ 10 R_{E}$ ). If the results presented in this paper are correct, then the cusp will extend over the complete front surface of the magnetosphere. The length of the cusp will thus be $\sim$ I $10 R_{E}=$ $2 \times 10^{5} \mathrm{~km}$ which results in a magnetopause cusp area of $\mathrm{dA}_{S}=1.3 \times 10^{9} \mathrm{~km}^{2}$. Using a value of $\mathrm{B}$ at $10 \mathrm{R}_{\mathrm{E}}$ of $\mathrm{B}_{\mathrm{S}}=50 \gamma$ (Fairfield, private communication, 1971) and $B_{I}=.5$ gauss at auroral heights the ratio of $\frac{B_{s}}{B_{I}}$ will be $10^{-3}$. Using the conversation of flux, $\mathrm{dA}_{I}$ (the cusp area at low altitudes) is given by

$$
\begin{aligned}
& \mathrm{dA}_{I}=\frac{B_{s}}{B_{I}} \cdot d_{s} \\
& d_{I}=1.3 \times 10^{6} \mathrm{~km}^{2}
\end{aligned}
$$

The longitudinal extent of magnetosheath fluxes reported in this paper is $\sim 4000 \mathrm{~km}$ which results in a latitudinal width of $\sim 320 \mathrm{~km}\left(3^{\circ}\right)$ at auroral heights which is in good agreement with the ISIS 1 observations. Frank (1970) also indicates that the cusp width does not increase more than a factor of 2 even for disturbed conditions. Everything else being the same, a factor of 2 increase in width at the magnetopause would result in a width of $\sim 1900 \mathrm{~km}$ at $3500 \mathrm{~km}$. This is also in good agreement with the maximum cusp width of $2000 \mathrm{~km}$ observed with ISIS 1 at this altitude on $8 \mathrm{June} 1969$, as mentioned earlier.

The difference in widths observed at low-altitudes by INJUN 5 and ISIS 1 could be the result of different instrumental sensitivities. Another, and more likely, possibility exists in the impression gained from a spectrogram presentation and the inferences made therefrom. Frank and Ackerson (1971) associated the sharp, low-energy burst at 23:31:00 UT 
(their plate 6a) with the low-altitude cusp. However if the low-latitude, low-altitude boundary of the cusp is associated with the sharp change (from 2000 to $150 \mathrm{ev)}$ in average energy at 23:30:05 UT (see Figure 16, Frank and Ackerson, 1971) and the upper boundary with the sharp burst at 23:31:00 UT, then the width of the cusp would be $-2^{\circ}$. This would result in good agreement between measurements made with ISIS 1 and INJUN 5 .

Frank (1971), in a recent letter, has indicated that his original estimate (Frank, 1970) of the low-altitude cusp width was much smaller than the average value of $200 \mathrm{~km}$ obtained from a larger set of IMP 5 data. This updated width brings the IMP 5 observations into much closer agreement with those observed by ISIS 1 and calculated in this section. However, this larger set of IMP 5 data still indicated two distinct, yet not mutually exclusive, field aligned 'sheets' of proton and electron fluxes (with electrons lying equatorward of protons) at mid altitudes in the cusp. No evidence of such clear separation can be found in the ISIS 1 data. Protons, if above the instrument threshold, are always coincident with electron fluxes. Electron fluxes in the cusp do have bursts superimposed on a background continuum flux but these electron tursts have no counterpart in the accompanying protion fluxes. Also no evidence for proton precipitation poleward of electron precipitation is observed in airborne photometric data (Eather and Mende, 1971b). If this sheet structure is a permanent feature of the cusp at mid-altitudes it then appears "chat "remixing" of the plasma must occur between $\sim 5 R_{E}$ and $1.5 R_{E}$. The resolution of this question should be possibly by a careful intercomparison of the available ground based and satellite data pertinent to the dayside magnetospheric cusps. 
CONCLUSIONS

From the data presented in this and earlier works by Winningham (1970), and Heikkila and Winningham (1971) the following conclusions are reached:

1. The long postulated free access of magnetosheath plasma to ionospheric heights does exist,

2. Access of magnetosheath plasma extends from 0800 to $1600 \mathrm{MLT}$ (magnetic local time) and is on the average $2^{\circ}$ to $3^{\circ}$ of invariant latitude wide at auroral heights,

3. Using the results of Fairfield (1968) penetration through the dayside magnetospheric cusps occurs over the complete front side of the magnetosphere and during disturbed periods possibly over a larger extent,

4. The postulated separation of proton and electron fluxes at mid-altitudes in the dayside cusps is not present at heights $\leq 1.5 \mathrm{R}_{E}$,

5. The dominant effect of increased magnetic activity is an equatorward motion of the boundary between open and closed field lines with the largest cusp width being a factor of 2 greater than the average,

6. The energy and number flux and particle average energies are sufficient to explain observed dayside auroral phenomena, and

7. Electron spectra observed concurrently at low and mid-altitudes in the cusp and outer zone are similar in shape and magnitude. 
REFERENCES

Eather, R. H., and Mende, S. B.: 1971a, J. Geophys. Res. 76, 1746.

Eather, R. H., and Mende, S. B.: 1971b, paper presented at Advanced Study Institute on Magnetosphere-Ionosphere Interactions, Dalseter, Norway.

Fatrfield, D. H.: 1968, I. Geophys. Res. 73, 7329.

Frank, L. A.: 1970, U. of Iowa Report, 70-55.

Frank, L. A.: 1971, J. Geophys. Res. 76, 2512.

Frank, L. A. and Ackerson, K. L.: 1971, J. Geophys. Res. 76, 3612.

Heikkila, W. J.: 1970 , J. Geophys. Res. $75,4877$.

Heikkila, W. J., Smith, J. B., Tarstrup, J., and Winningham, J. D.: 1970, Rev. Sci. Instrum. 41, 1393.

Heikkila, W. J., and Winningham, J. D.: 1971, J. Geophys. Res. $76,883$.

Rees, M. H.: 1964, Planet. Space Sci. $12,722$.

Russell, C. T., Chappeli, C. R., Montgomery, M. D., Neugebauer, M., and Scarf, F. L.: 1971; submitted to J. Geophys. Res.

Winningham, J. D.: 1970, Dissertation, Texas A \& M University. 
FIGURE CAPTIONS

Figure 1a.

Figure Ib.

Figure 2 .

Figure 3.

Figure 4.

Figure 5.

Figure 6.

Figure 7.
Electron spectrogram for 28 Apr11 1969 at 19:50:53 UT.

Proton spectrogram for 28 April 1969 at 19:50:53 UT.

Electron and proton differential spectra for the data contained in Figure $1 \mathrm{a}$ and $\mathrm{b}$. Cusp spectra were obtained for the period 19:51:57 to 19:52:07 UT. The outer zone spectrum is for 19:54:01 UT.

Frequency of occurrence for the. peak energy of the primary electron spectrum in the dayside magnetospherlc cusp. Normalized proton and electron pitch angle distributions for the energy range 73 to $420 \mathrm{ev}$. These distributions are for the period 19:51:57 to 19:52:07 UT in Figure 1. Unity represents $2.5 \times 10^{8} \mathrm{~cm}^{-2}$ ster $\mathrm{sec}^{-1}$ and $6.0 \times 10^{-2}$ ergs $\mathrm{cm}^{-2}$ ster $^{-1} \mathrm{sec}^{-1}$ for electrons; and $1.0 \times 10^{7} \mathrm{~cm}^{-2}$ ster $^{-1}$ $\sec ^{-1}$ and $3.0 \times 10^{-2}$ ergs $\mathrm{cm}^{-2}$ ster $^{-1} \mathrm{sec}^{-1}$ for protons. Electron spectra for the data contained in Figure 4. Extent of magnetosheath plasma penetration in magnetic local time and invariant latitude as a function of $k_{p}$. Comparison of outer zone and cusp spectra obtained within 2 hours of local and universal time on $11 \mathrm{July} 1969$ with ISIS 1 and IMP 5 . 


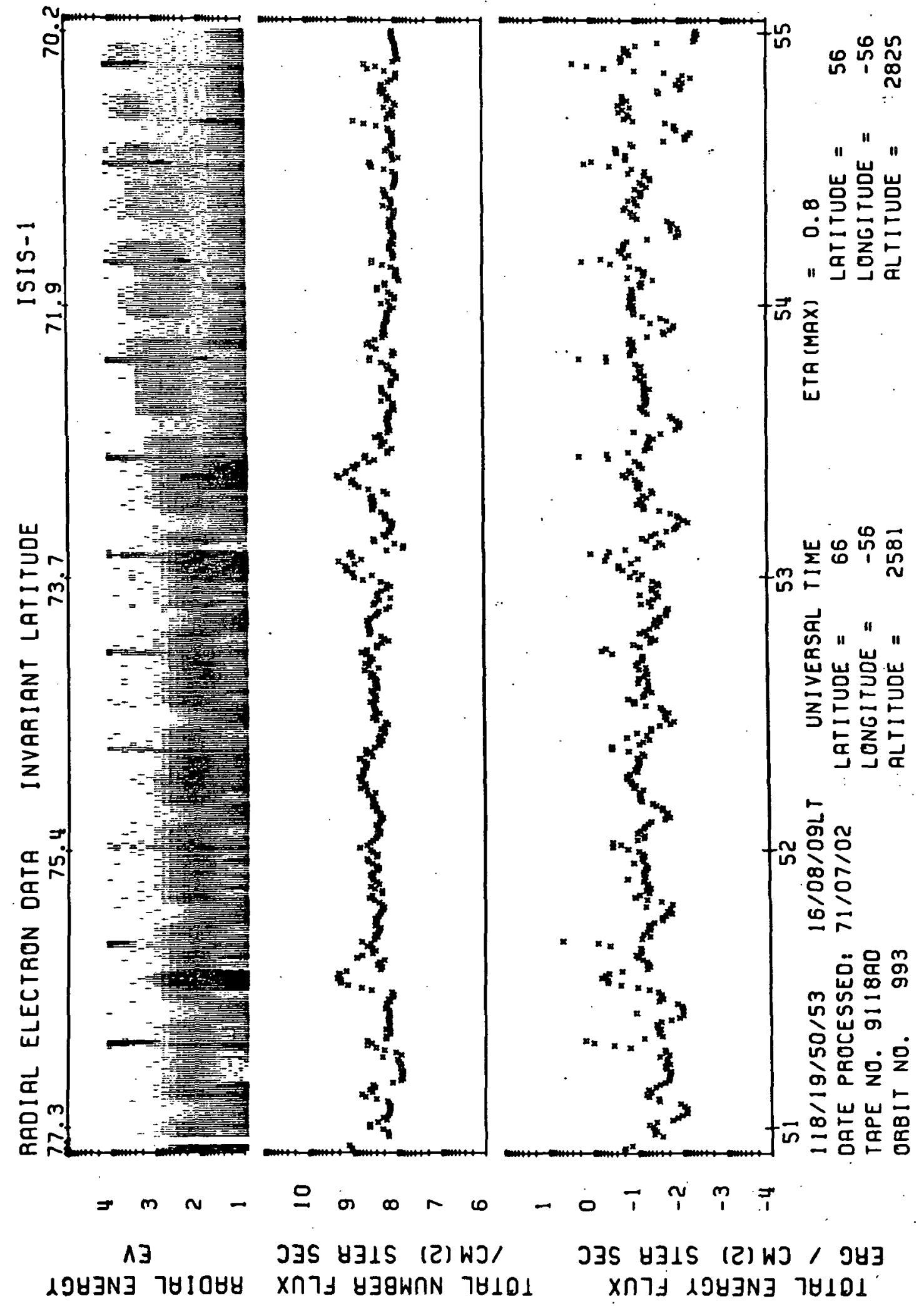

Figure 1a. 


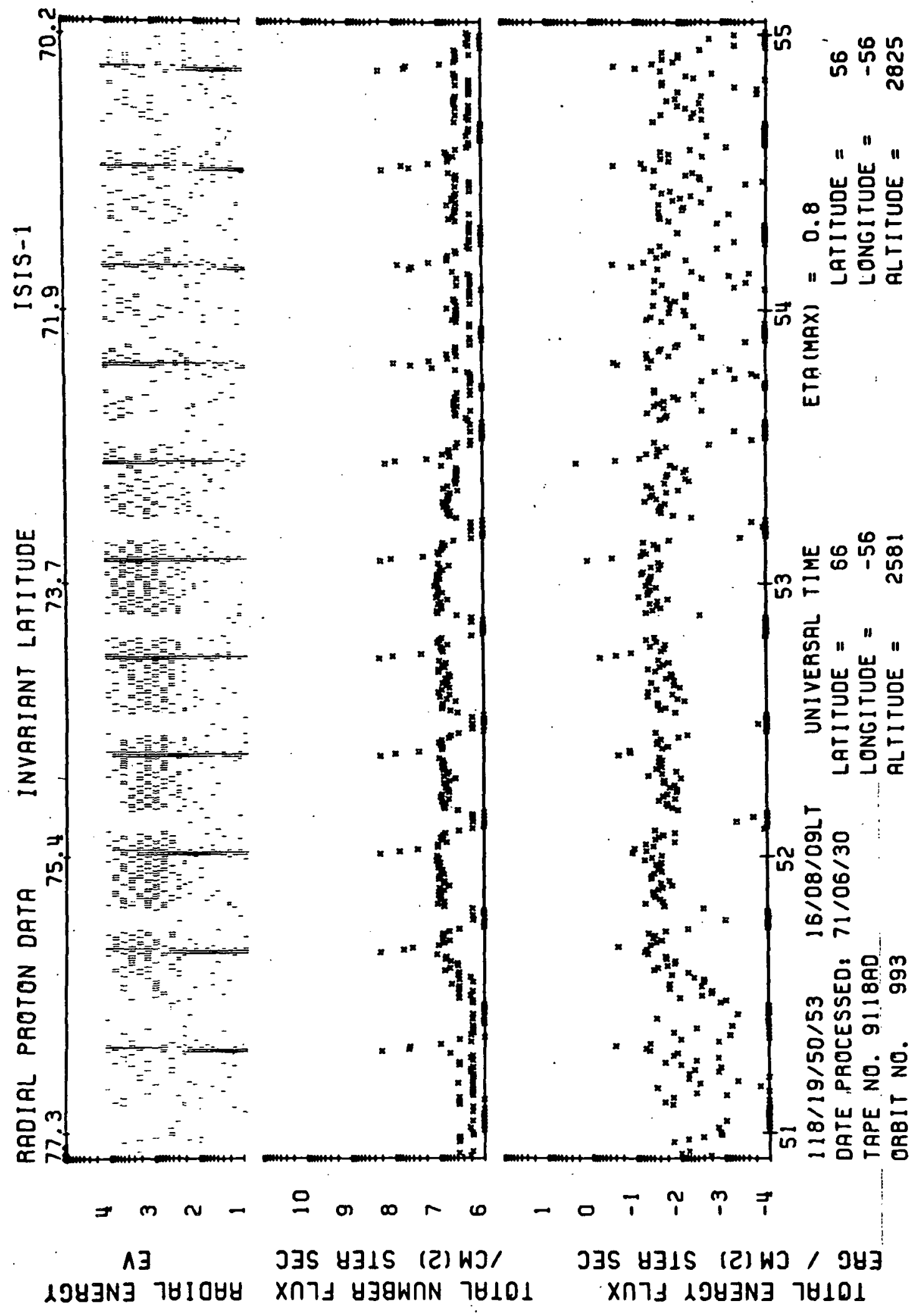

Figure ib. 


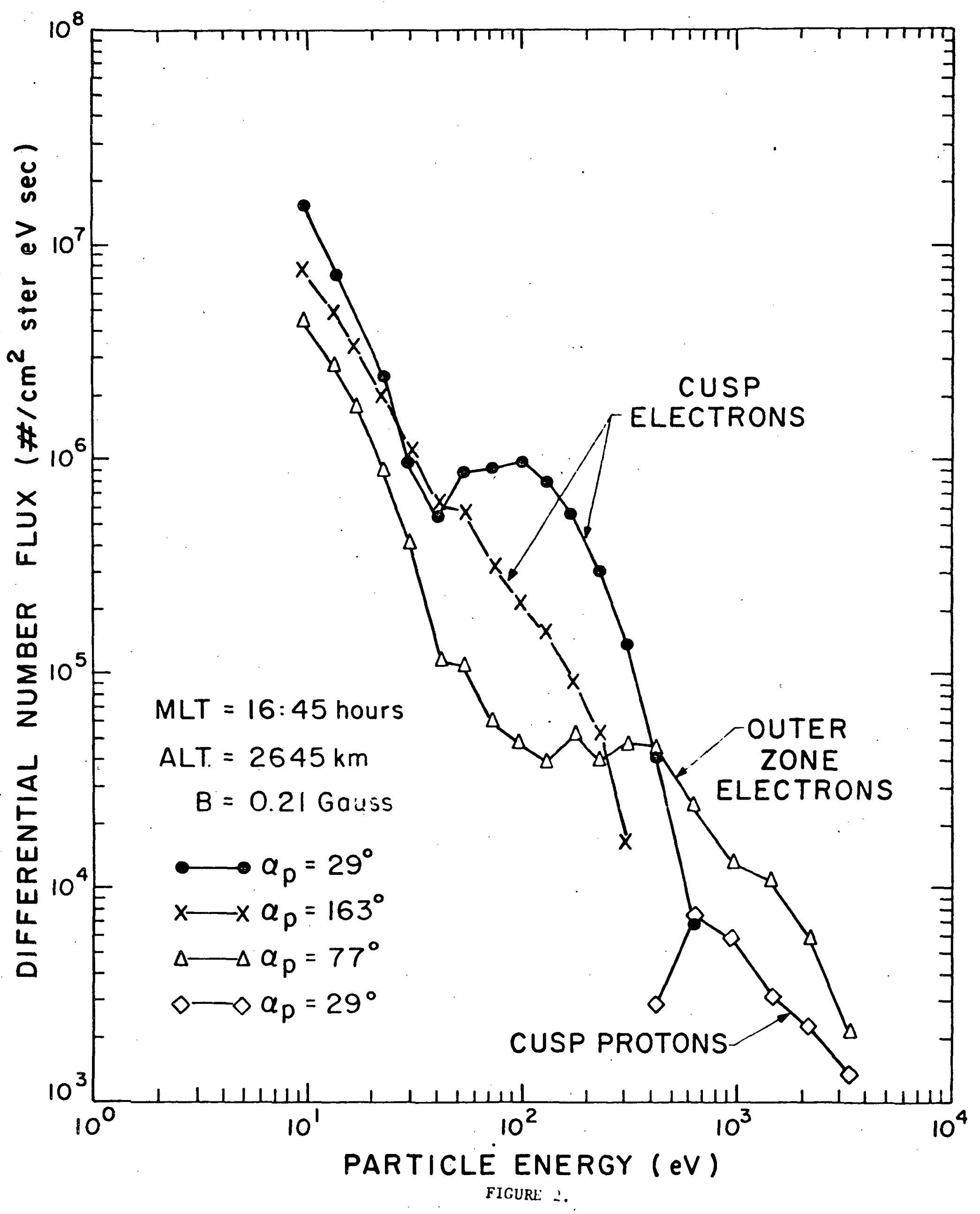




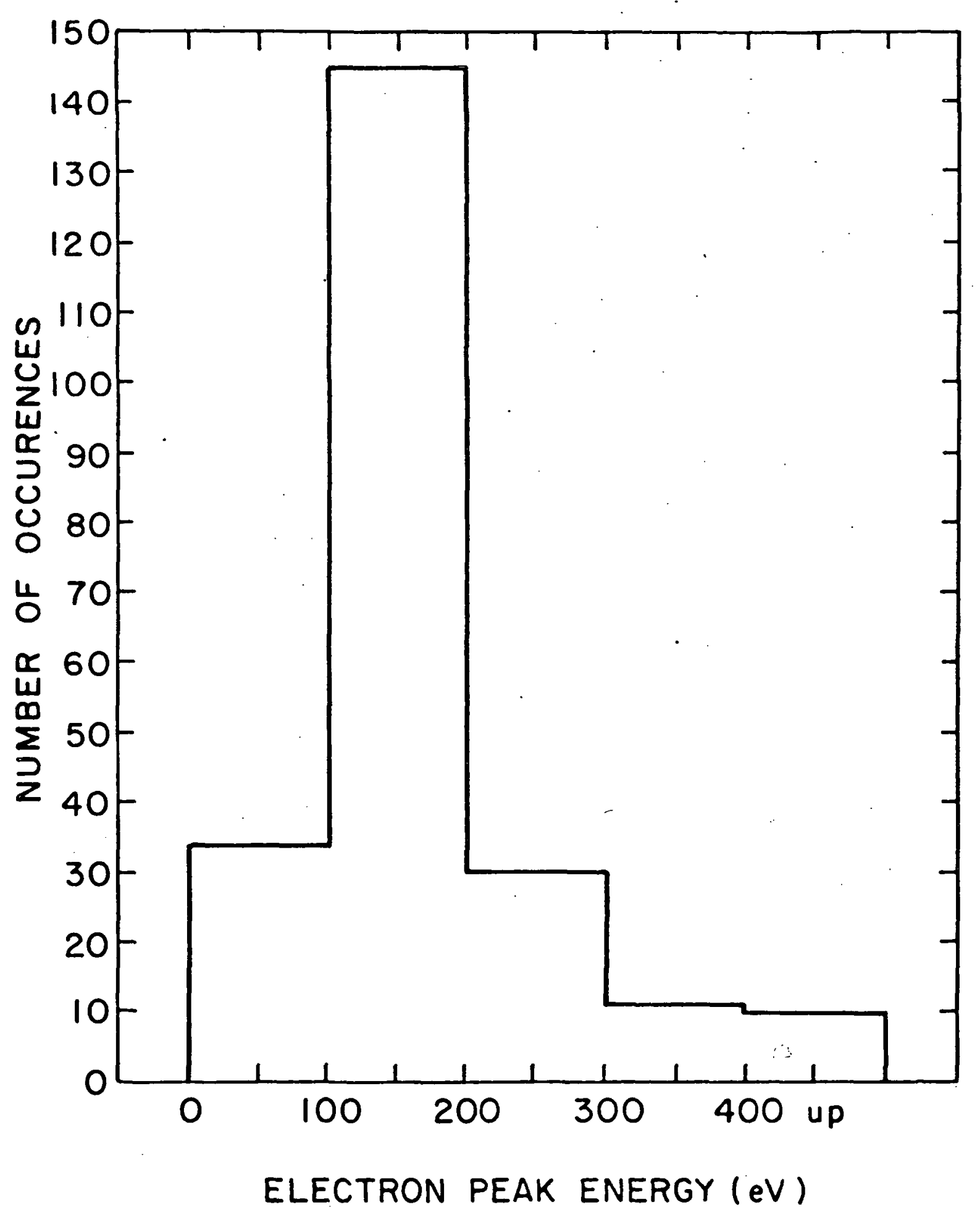

FIGURE 3. 


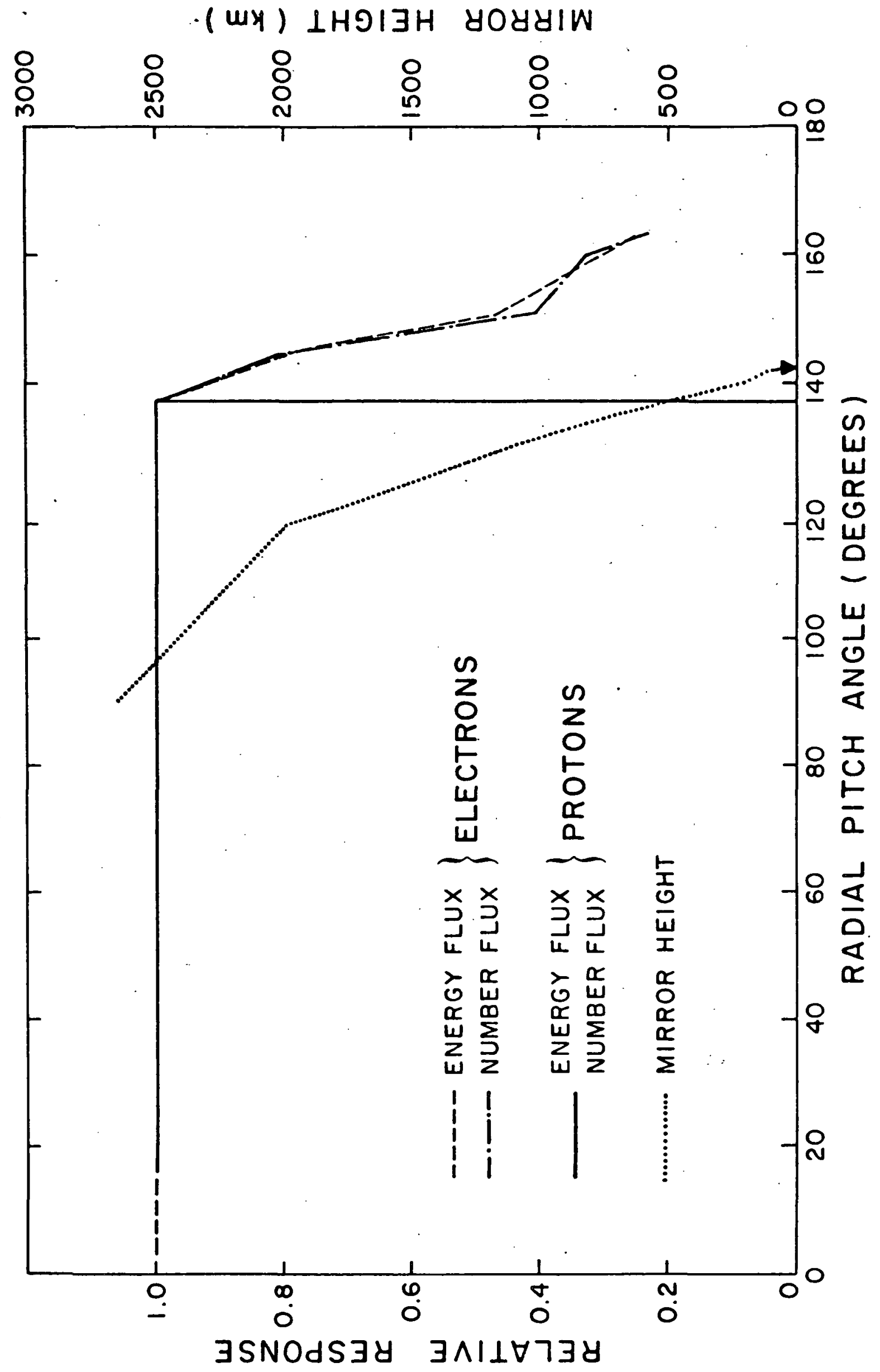

FIGURE 4. 


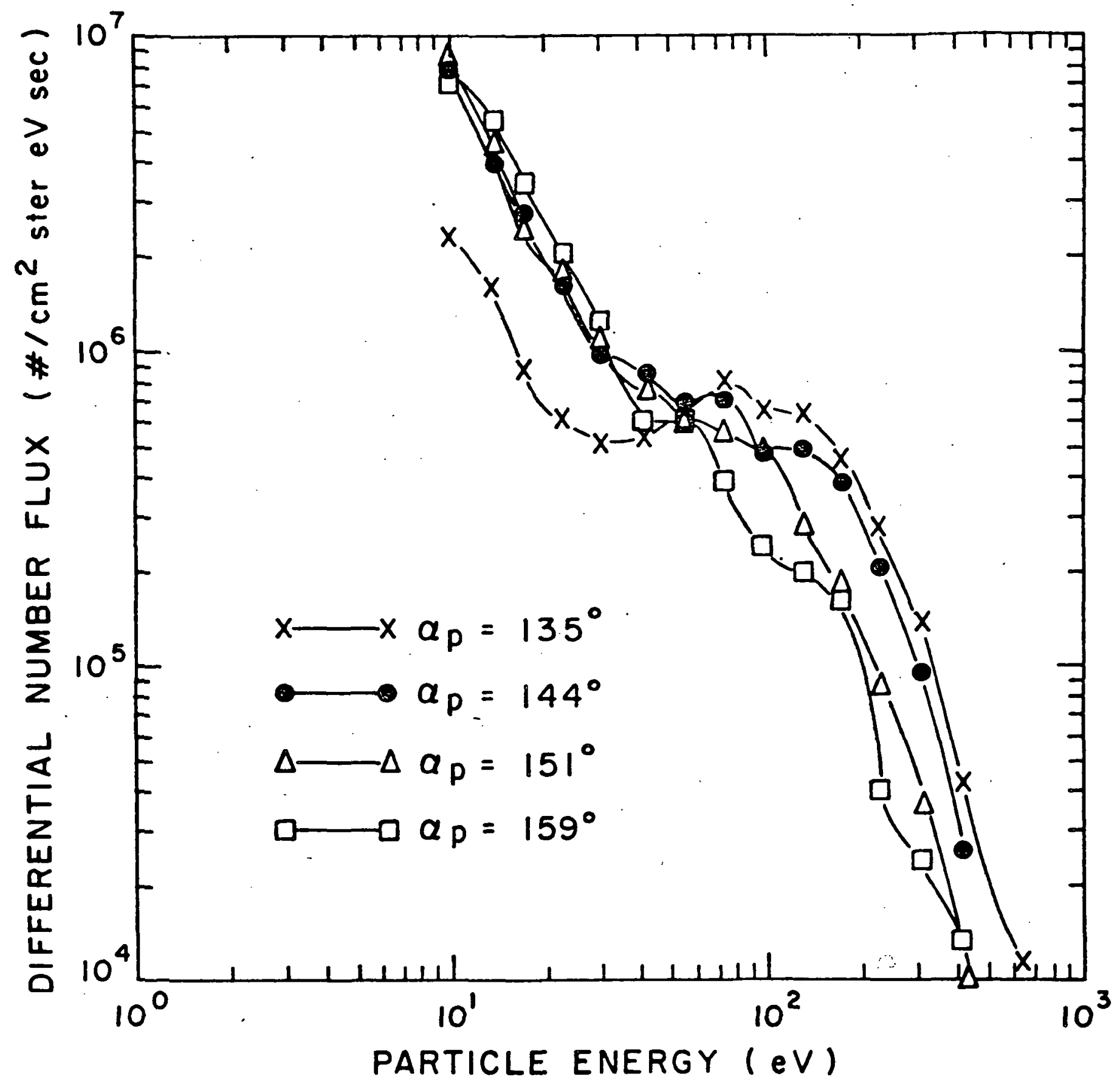

FIGURE 5. 


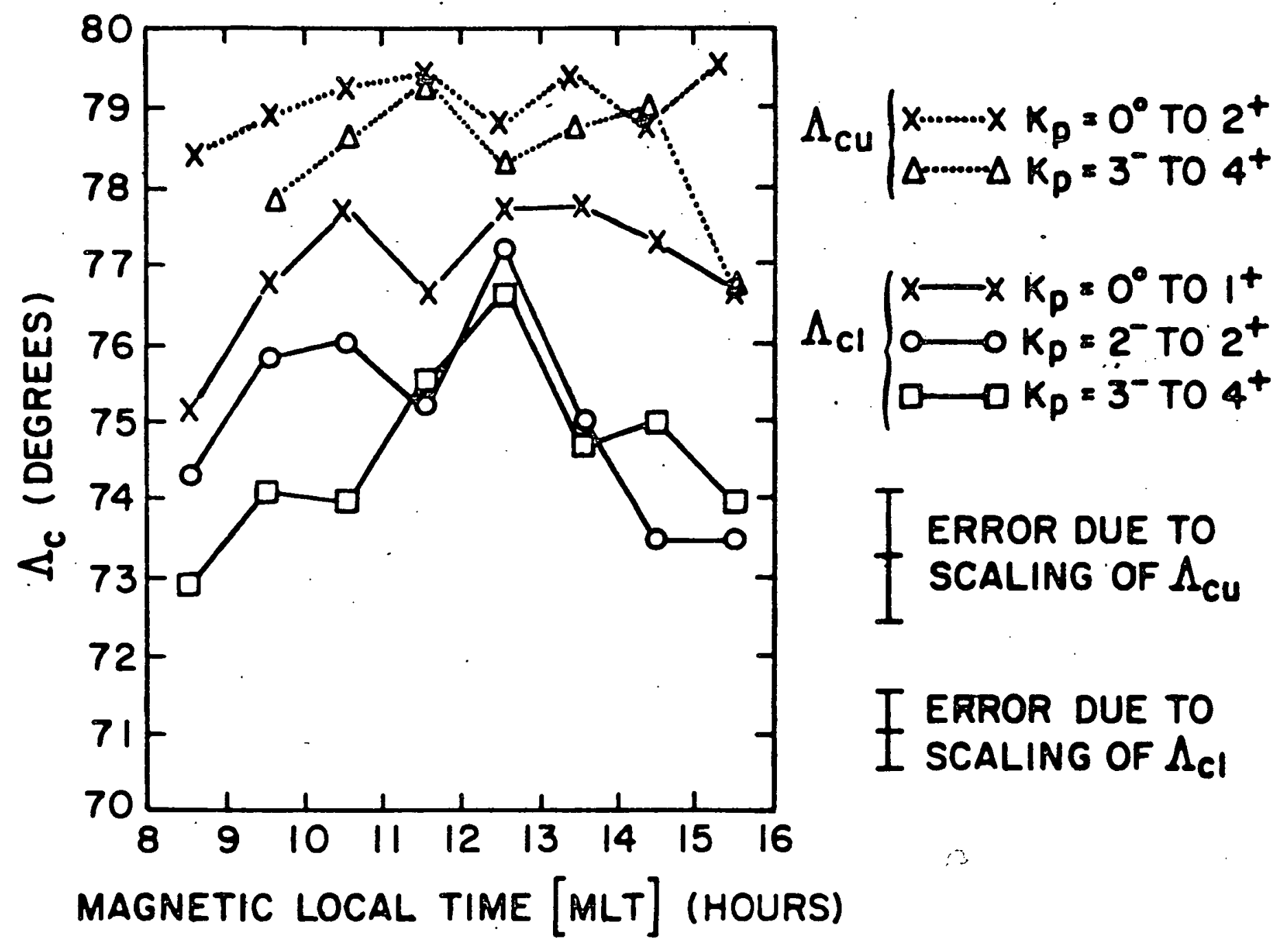

FIGURE 6. 


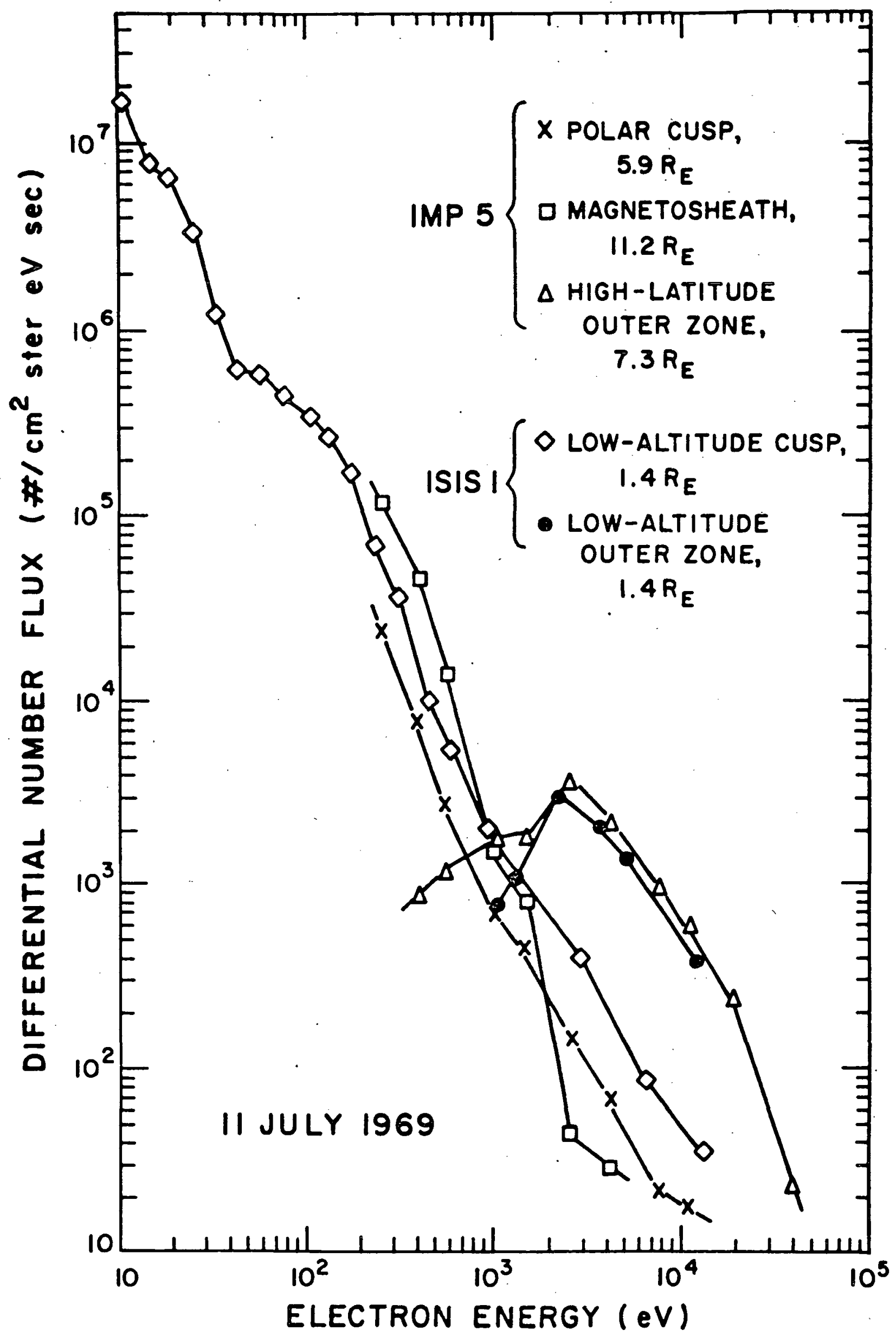

FIGURE 7. 